\title{
The Quotient Construction for a Class of Compact Einstein Manifolds
}

\author{
Brett McInnes \\ Department of Mathematics, National University of Singapore, 10 Kent Ridge Crescent, 0511 \\ Republic of Singapore
}

Received March 1, 1992; in revised form October 5, 1992

\begin{abstract}
Given any Einstein manifold $M^{E}$, one can obtain further examples of Einstein manifolds by taking the quotient $M^{E} / G$ by a freely acting, properly discontinuous group of isometries. We study this method in the case in which $M^{E}$ is Kählerian, $M^{E} / G$ is compact, and the Ricci curvature is non-negative. In many cases, the candidates for $G$ can be completely classified.
\end{abstract}

\section{Introduction}

The Einstein manifolds constitute perhaps the most interesting special class of Riemannian manifolds, and their properties have frequently attracted the attention of physicists: one thinks of the application of Myers' theorem by Freund and Rubin [5], of Yau's theorem by Candelas et al. [3], of hyperkähler geometry by workers in supersymmetric sigma models [8], and so on. A more complete understanding of the full range of Einstein manifolds would clearly be highly desirable both in physics and in mathematics [2].

Given any Einstein manifold $M^{E}$, one has a canonical procedure for constructing further examples of the same dimension. If a group $G$ acts isometrically, freely, and properly discontinuously on $M^{E}$, then $M^{E} / G$ is also an Einstein manifold. The distinction between $M^{E}$ and $M^{E} / G$ is purely global, but this global distinction can have physical consequences: in string theory, the fact that Calabi-Yau manifolds of the form $M^{E} / G$ can support flat gauge fields with non-trivial holonomy is the basis of the "Hosotani mechanism" for breaking gauge symmetries.

The "quotient Calabi-Yau" manifolds of string theory are special examples of an interesting class of manifolds which we may call the "locally Kählerian" Einstein manifolds. These are Einstein manifolds of the form $M^{E} / G$, where $M^{E}$ is a simply connected, irreducible Kähler-Einstein manifold, and where $G$ acts isometrically but not necessarily holomorphically (so that the restricted holonomy group of $M^{E} / G$ is contained in $U(n)$, where $n$ is the complex dimension of $M^{E}$ ). In this work we present some results on this important class of Einstein manifolds, mainly confining ourselves to the case in which $M^{E} / G$ is compact and of non-negative Ricci 
curvature. In particular, we find that the candidates for $G$ can be completely classified for many types of the manifold $M^{E}$.

\section{The Structure of $G$}

Recall first that if $M^{E} / G$ is compact and locally irreducible, then [4] $M^{E}$ must be compact if the Ricci curvature is non-negative. Thus $G$ must be finite. Now if $M^{E}$ is a compact irreducible Kähler-Einstein manifold with non-negative Ricci curvature, then its holonomy group is either $\operatorname{Sp}\left(\frac{1}{2} n\right)$ (if the complex dimension $n=\operatorname{dim}_{\mathbb{C}}\left(M^{E}\right)$ is even), $S U(n)$, or $U(n)$. We consider the three cases in turn.

Case [1]. $M^{E}$ is Hyperkählerian, with Holonomy $\operatorname{Sp}\left(\frac{1}{2} n\right)$. We denote such a manifold by $S^{H}$. These manifolds possess a two-sphere of parallel complex structures, giving rise to a two-sphere of Kähler forms, $\left\{a \phi_{I}+b \phi_{J}+c \phi_{K}\right\}$, where $a, b, c$ are real numbers such that $a^{2}+b^{2}+c^{2}=1$. If $V$ is the vector space spanned over the reals by $\phi_{I}, \phi_{J}, \phi_{K}$, then the hyperkähler metric induces a canonical inner product on $V$, and then the two-sphere of Kähler forms can be regarded as the unit two-sphere in $V$.

Let $f: S^{H} \rightarrow S^{H}$ be any (not necessarily holomorphic) isometry of $S^{H}$, and let $a \phi_{I}+b \phi_{J}+c \phi_{K}$ be any element of $V$. If $\nabla$ is the Levi-Civitá connection of the hyperkähler metric, then

$$
\nabla\left[f^{*}\left(a \phi_{I}+b \phi_{J}+c \phi_{K}\right)=f^{*} \nabla\left(a \phi_{I}+b \phi_{J}+c \phi_{K}\right)=0 .\right.
$$

But [2] all parallel forms on $S^{H}$ belong to the exterior algebra generated over the reals by $\phi_{I}, \phi_{J}, \phi_{K}$. Thus $f^{*}$ is a linear automorphism of $V$. Because $f$ is an isometry, the restriction of $f^{*}$ to the two-sphere of Kähler forms is a linear isometry of the two-sphere. This simple construction yields the following information on the isometry group of $S^{H}$ and on its freely acting subgroups.

Theorem 1. Let $S^{H}$ be a connected, compact, irreducible hyperkähler manifold. Then

[a] The full isometry group, $\operatorname{Isom}\left(S^{H}\right)$, is a group extension of a finite subgroup of the orthogonal group $O(3)$ by a finite group of symplectomorphisms. (A symplectomorphism of a hyperkähler manifold is a holomorphic map which preserves the canonical non-degenerate parallel holomorphic two-form.)

[b] If $G$ is a non-trivial freely acting subgroup of $\operatorname{Isom}\left(S^{H}\right)$, then $G$ is a finite subgroup of $S O(3)$, of order and isomorphism type governed by $\operatorname{dim}_{\mathbb{C}}\left(S^{H}\right)$ : to be precise,

(i) If $\operatorname{dim}_{\mathbb{C}}\left(S^{H}\right)$ is a multiple of four, then $G$ is a finite cyclic group of order dividing $1+\frac{1}{2} \operatorname{dim}_{\mathbb{C}}\left(S^{H}\right)$.

(ii) Otherwise, $G$ is either as in (i), or $G$ is a finite dihedral or polyhedral group of order dividing $2+\operatorname{dim}_{\mathbb{C}}\left(S^{H}\right)$.

Proof. [a] By Bochner theory and the compactness of $S^{H}$, $\operatorname{Isom}\left(S^{H}\right)$ is necessarily finite [9]. Let $F$ be the homomorphism from $\operatorname{Isom}\left(S^{H}\right)$ into $O(3)$ defined by

$$
F: f \rightarrow\left(f^{-1}\right)^{*},
$$

where $\left(f^{-1}\right)^{*}$ is restricted to the two-sphere of Kähler forms discussed above. The image of $F$ is some finite subgroup of $O(3)$ : that is, a cyclic, dihedral, or polyhedral group, or some product of one of these with $\mathbb{Z}_{2}$. The kernel of $F$ consists of 
isometries satisfying

$$
f^{*} \phi_{I}=\phi_{I}, \quad f^{*} \phi_{J}=\phi_{J}, \quad f^{*} \phi_{K}=\phi_{K} .
$$

Such an isometry is holomorphic (with respect to every complex structure in the two-sphere). Now set $\omega=\phi_{J}+i \phi_{K}$. Then $\omega$ is a closed holomorphic two-form with respect to $I$. Since $S^{H}$ is irreducible, we can take $\omega$ to be the (essentially unique) complex symplectic form on $S^{H}$. Clearly $\operatorname{Ker}(F)$ consists of symplectomorphisms. Now Isom $\left(S^{H}\right) / \operatorname{Ker}(F)$ is isomorphic to a finite subgroup of $O(3)$, and this completes the proof of part [a].

[b] According to Beauville [1], the only non-trivial Dolbeault cohomology groups of $S^{H}$ are those generated by the canonical holomorphic two-form. It follows that every symplectomorphism has a positive holomorphic Lefschetz number [6]. Since $G$ acts freely, and since $\operatorname{Ker}(F)$ consists of symplectomorphisms, the holomorphic Lefschetz fixed point formula [6] implies that $G$ and $\operatorname{Ker}(F)$ intersect only in the identity map; and so the restriction of $F$ to $G$ maps the latter isomorphically onto some finite subgroup of $O(3)$. In fact, the image lies in $S O(3)$. To see this, let $f$ be in $G$, and let $\mathbb{Z}_{m}$ be the cyclic group generated by $f$. According to Reference [10], there is a complex structure $I$ on $S^{H}$ such that $\mathbb{Z}_{m}$ acts holomorphically with respect to $I$. As $f$ is both isometric and $I$-holomorphic, it preserves $\phi_{I}$. That is, $f^{*}$ fixes some directed axis of the two-sphere of Kähler forms. Now as before, we take $\phi_{J}+i \phi_{K}$ to be the complex symplectic form; the irreducibility of $S^{H}$ implies that we have

$$
f^{*}\left(\phi_{J}+i \phi_{K}\right)=\alpha\left(\phi_{J}+i \phi_{K}\right)
$$

where, by the maximum principle and the compactness of $S^{H}, \alpha$ is a constant complex number. As $f$ is of finite order, it now follows that $\alpha$ is unimodular, and so $f^{*}$ merely rotates the two-sphere of Kähler forms through the corresponding angle. Thus $G$ contains no reflections and is a subgroup of $S O(3)$.

The cyclic group generated by $f$ acts freely and holomorphically. By the Riemann-Roch-Hirzebruch theorem [2], the order of this cyclic group must divide the arithmetic genus of $S^{H}$, which is $1+\frac{1}{2} \operatorname{dim}_{\mathbb{C}}\left(S^{H}\right)$ by the work of Beauville [1]. Thus $G$ must be a cyclic, dihedral, or polyhedral group such that the order of each element divides this integer. (Of course, it is clear from their action on the two-sphere of Kähler forms that the dihedral and polyhedral groups cannot act holomorphically with respect to any one complex structure; however, for each element, one can find such a complex structure.) If $\operatorname{dim}_{\mathbb{C}}\left(S^{H}\right)$ is a multiple of four, this is impossible for the dihedral and polyhedral groups, all of which contain at least one element of order two. Hence the odd cyclic groups are the only possibilities in this case. Otherwise, it is a straightforward exercise in finite group theory to show that the least common multiple of the orders of the elements of the dihedral group $D_{2 m}$ (order $\left.2 m\right)$ is $2 m$ if $m$ is odd, $m$ if $m$ is even. For the polyhedral groups $P_{12}$ (tetrahedral), $P_{24}$ (octahedral), and $P_{60}$ (icosahedral), the corresponding numbers are 6,12 , and 30 . Thus, in all these cases, the order of the group must divide $2+\operatorname{dim}_{\mathbb{C}}\left(S^{H}\right)$. This completes the proof.

This theorem contains, in the case $\operatorname{dim}_{\mathbb{C}}\left(S^{H}\right)=2$, a well-known result of Hitchin [7]: this states that a compact Riemannian 4-manifold, locally isometric to a $K 3$ surface endowed with a Ricci-flat Kähler metric, must have the structure $K 3 / \mathbb{Z}_{2}^{+}$or $K 3 /\left[\mathbb{Z}_{2}^{+} \times \mathbb{Z}_{2}^{-}\right]$, where $\mathbb{Z}_{2}^{+}$is generated by a fixed point free holomorphic involution, and $\mathbb{Z}_{2}^{-}$by a fixed point free antiholomorphic involution. For in this case $K 3$ is a hyperkähler manifold, $2+\operatorname{dim}_{\mathbb{C}}(K 3)=4$, and $\mathbb{Z}_{2} \times \mathbb{Z}_{2}$ is the 
dihedral group of order four. Thus, for example, the generalisation of Hitchin's theorem to complex dimension 6 states that the only manifolds locally isometric to a compact irreducible hyperkähler manifold of that dimension are of the form $S^{H} / \mathbb{Z}_{2}, S^{H} / \mathbb{Z}_{4}, S^{H} /\left[\mathbb{Z}_{2} \times \mathbb{Z}_{2}\right]$, or $S^{H} / D_{8}$.

Case [2]. $M^{E}$ has Holonomy $S U(n)$. We denote such a manifold by $S^{C Y}$ and describe it as a Calabi-Yau manifold. These manifolds do not, of course, possess a twosphere of parallel complex structures, and in fact each isometry is either holomorphic or antiholomorphic with respect to a fixed complex structure.

Theorem 2. Let $S^{\mathrm{CY}}$ be a compact, connected, simply connected, irreducible CalabiYau manifold. Then

[a] The full isometry group, $\operatorname{Isom}\left(S^{\mathrm{CY}}\right)$, is a group extension of a finite cyclic or dihedral group by a finite group which is isomorphic to a group of holomorphic maps which preserve the complex volume form on $S^{\mathrm{CY}}$.

[b] If $G$ is a non-trivial freely acting subgroup of $\operatorname{Isom}\left(S^{\mathrm{CY}}\right)$, then

(i) If $\operatorname{dim}_{\mathbb{C}}\left(S^{\mathrm{CY}}\right)$ is even, $G$ is isomorphic to $\mathbb{Z}_{2}^{+}$, to $\mathbb{Z}_{2}^{-}$, or to $\mathbb{Z}_{2}^{+} \times \mathbb{Z}_{2}^{-}$, where $\mathbb{Z}_{2}^{+}\left(\mathbb{Z}_{2}^{-}\right)$is generated by an (anti) holomorphic involution.

(ii) If $\operatorname{dim}_{\mathbb{C}}\left(S^{\mathrm{CY}}\right)$ is odd, then either $S^{\mathrm{CY}} / G$ is itself a Calabi-Yau manifold, or it has the structure $M^{\mathrm{CY}} / \mathbb{Z}_{2}^{-}$, where $M^{\mathrm{CY}}$ is a Calabi-Yau manifold covered by $S^{\mathrm{CY}}$, and $\mathbb{Z}_{2}^{-}$is generated by an antiholomorphic involution.

Proof. Let $\delta$ denote the complex volume form. Then with an appropriate normalisation we can put

$$
\bar{\delta} \wedge \delta=\phi^{n},
$$

where $\phi$ is the Kähler form. As $\delta$ is unique modulo complex scalars, we have $f^{*} \delta=c \delta$, where $c$ is constant, and where $f$ is a holomorphic isometry. Such an isometry must be orientation-preserving, and so $c$ must satisfy $\bar{c} c=1$. Similarly, if $f$ is an antiholomorphic isometry, we have $f^{*} \phi=-\phi$ and so if we put $f^{*} \delta=c \bar{\delta}$, then

Thus $\bar{c} c=1$, and so

$$
\begin{aligned}
f^{*}(\bar{\delta} \wedge \delta) & =\bar{c} c \delta \wedge \bar{\delta}=(-1)^{n} \bar{c} c \bar{\delta} \wedge \delta=f^{*} \phi^{n} \\
& =(-1)^{n} \bar{\delta} \wedge \delta .
\end{aligned}
$$

$$
\left(f^{2}\right)^{*} \delta=c \bar{c} \delta=\delta .
$$

Hence $f$ is represented as an involution when acting on $\delta$.

Now as in Theorem $1, \operatorname{Isom}\left(S^{\mathrm{CY}}\right)$ is finite. Thus the homomorphism $F: f \rightarrow\left(f^{-1}\right)^{*}$, where $\left(f^{-1}\right)^{*}$ is restricted to its action on $\delta$, represents any group of holomorphic isometries as a finite cyclic group. If a group of isometries contains an antiholomorphic element $h$, with $h^{*} \delta=b \bar{\delta}$, then $F$ maps $h$ to an involution which satisfies

$$
h^{*} f^{*} h^{*} \delta=b h^{*} f^{*} \bar{\delta}=b \bar{c} h^{*} \bar{\delta}=b \bar{b} \bar{c} \delta=\bar{c} \delta=\left(f^{-1}\right)^{*} \delta,
$$

where $f$ is a holomorphic isometry. Thus the image of $F$ is either a finite cyclic group if Isom $\left(S^{\mathrm{CY}}\right)$ consists only of holomorphic maps, or a finite dihedral group if it contains antiholomorphic elements. The kernel consists of holomorphic maps satisfying $f^{*} \delta=\delta$. Thus $\operatorname{Isom}\left(S^{\mathrm{CY}}\right)$ must be as described.

[b] Let $\operatorname{dim}_{\mathbb{C}}\left(S^{\mathrm{CY}}\right)$ be even. Since $\delta$ is essentially the only holomorphic form on $S^{\mathrm{CY}}$, we can readily compute the holomorphic Lefschetz number [6] of any element of 
the kernel of the homomorphism $F$ defined above. The only non-zero trace is on forms of type $(0, n)$, and it is given by

$$
\text { Trace }\left.f^{*}\right|_{H_{\bar{i}}^{0, n}\left(S^{\mathrm{CY}}\right)}=\int_{S^{\mathrm{CY}}} \delta \wedge f^{*} \bar{\delta} / \int_{S^{\mathrm{CY}}} \delta \wedge \bar{\delta}=1 .
$$

Thus the holomorphic Lefschetz number of any element of the kernel of $F$ is 2, and so, by the holomorphic Lefschetz fixed point formula, it follows that every element of this kernel has a fixed point. Thus if $G$ is a freely acting subgroup of $\operatorname{Isom}\left(S^{C Y}\right)$, then $G$ intersects this kernel only in the identity. Thus $G$ must itself be a cyclic or dihedral group. Assume that $G$ is cyclic, generated by a holomorphic map $f$. Since $f$ has no fixed point, its holomorphic Lefschetz number must be zero, and so we must have $f^{*} \delta=-\delta$. Clearly $G=\mathbb{Z}_{2}^{+}$in this case. Assume instead that $G$ is dihedral. Then its cyclic normal subgroup of index 2 must be either trivial or isomorphic to $\mathbb{Z}_{2}^{+}$, and so $G=\mathbb{Z}_{2}^{-}$or $\mathbb{Z}_{2}^{+} \times \mathbb{Z}_{2}^{-}$, as claimed.

If $\operatorname{dim}_{\mathbb{C}}\left(S^{\mathrm{CY}}\right)$ is odd, then the holomorphic Lefschetz fixed point formula requires that each holomorphic $f$ with no fixed points must satisfy $f^{*} \delta=\delta$. Thus the homomorphism $F$ maps $G$ onto either $\mathbb{Z}_{1}$ or $\mathbb{Z}_{2}$. In the first case, $G$ acts holomorphically and preserves $\delta$, which therefore projects down to the quotient: therefore $S^{\mathrm{CY}} / G$ is a compact complex manifold with no holomorphic forms other than the projected complex volume form, which is parallel with respect to the projected metric. That is, the quotient $S^{\mathrm{CY}} / G$ is a Calabi-Yau manifold. (Notice that this never occurs when the complex dimension is even: the manifolds of the form $S^{\mathrm{CY}} / G$ are not Calabi-Yau manifolds in that case if $G$ is non-trivial.) In the other case, when the image is $\mathbb{Z}_{2}, G$ contains a normal subgroup $N$ of index 2 , and $N$ acts freely, holomorphically, and preserves the complex volume form. Thus $M^{\mathrm{CY}}=S^{\mathrm{CY}} / N$ is a Calabi-Yau manifold, and since $G / N=\mathbb{Z}_{2}$, we have

$$
S^{\mathrm{CY}} / G=M^{\mathrm{CY}} / \mathbb{Z}_{2},
$$

where $\mathbb{Z}_{2}$ is generated by a fixed point free antiholomorphic involution on $M^{\mathrm{CY}}$. This completes the proof.

Case [3]. Let $M^{E}$ have Positive First Chern Class. This is the simplest case.

Theorem 3. Let $S^{P}$ be a compact, irreducible, connected Kähler-Einstein manifold of positive scalar curvature, and let $G$ be a finite freely acting non-trivial group of isometries of $S^{P}$. Then $G$ is isomorphic to $\mathbb{Z}_{2}^{-}$, generated by an antiholomorphic involution.

Proof. By Bochner theory [9], $S^{P}$ has no non-trivial holomorphic forms. The Lefschetz fixed point formula implies that every holomorphic map on $S^{P}$ has a fixed point. Thus every element of $G$ other than the identity must be antiholomorphic. As the product of two antiholomorphic maps is holomorphic, this means that $G=\mathbb{Z}_{2}^{-}$.

The full range of compact Einstein manifolds which can be obtained by applying the quotient construction to Kähler manifolds may be classified as follows.

Theorem 4. Let $M^{E} / G$ be an Einstein manifold, where $M^{E}$ is a compact, connected, simply connected, irreducible Kähler manifold, and $G$ is a non-trivial, finite, freely acting group of isometries. Then $M^{E} / G$ belongs to one of the following three classes:

[a] $M^{E} / G$ is itself a projective-algebraic Kähler-Einstein manifold.

[b] $M^{E} / G$ is the quotient of a manifold of type [a] by $\mathbb{Z}_{2}^{-}$, generated by an antiholomorphic involution. 
[c] $M^{E} / G$ has the form $S^{H} / P_{12}$, or $S^{H} / P_{24}$, or $S^{H} / P_{60}$, where $S^{H}$ is a compact hyperkähler manifold and $P_{12}, P_{24}, P_{60}$ are the polyhedral groups.

Proof. If $G$ acts holomorphically on $M^{E}$, then $M^{E} / G$ is Kähler-Einstein and $M^{E}$ is projective, by means of a straightforward application of the Kodaira embedding theorem [6]. Hence $M^{E} / G$ is projective.

If $G$ does not act holomorphically, then unless $M^{E}$ is hyperkählerian, each element of $G$ must be either holomorphic or antiholomorphic with respect to some fixed complex structure. In the hyperkählerian case, the same statement holds true when $G$ is cyclic or dihedral. In all these cases, $G$ has a normal subgroup of index 2 which acts holomorphically, and so we have parts [a] and [b]. (For example, the locally hyperkählerian manifolds of the form $S^{H} / D_{2 m}$ can be expressed as $\left(S^{H} / \mathbb{Z}_{m}\right) / \mathbb{Z}_{2}$, where $\mathbb{Z}_{m}$ is the cyclic normal subgroup of index 2 in $D_{2 m}$. ) The only remaining cases are given in part [c]. This completes the proof.

Notice that, in this theorem, it is not necessary to assume that the scalar curvature is non-negative: that is, the theorem classifies all quotients of compact irreducible Kähler-Einstein manifolds.

\section{Conclusion}

There are many examples of compact Einstein manifolds which can be obtained from Kähler manifolds by means of the quotient construction. These, however, are most naturally discussed in the context of an analysis and classification of their holonomy groups. In fact, the Kähler-Einstein quotient construction plays a central role in the holonomy theory of non-simply-connected compact Riemannian manifolds. For an application of the latter in physics, see Reference [11].

\section{References}

1. Beauville, A.: Variétés Kahleriennes dont la première classe de Chern est nulle. J. Diff. Geom. 18, 755-782 (1983)

2. Besse, A.: Einstein manifolds. Berlin, Heidelberg, New York: Springer 1987

3. Candelas, P., Horowitz, G.T., Strominger, A., Witten, E.: Vacuum configurations for superstrings. Nucl. Phys. B 258, 46-74 (1985)

4. Cheeger, J., Gromoll, D.: On the structure of complete manifolds of non-negative curvature. Ann. Math. 96, 413-443 (1972)

5. Freund, P.G.O., Rubin, M.A.: Dynamics of dimensional reduction. Phys. Lett. B97, 233-235 (1980)

6. Griffiths, P., Harris, J.: Principles of algebraic geometry. New York: Wiley 1978

7. Hitchin, N.: Compact four-dimensional Einstein manifolds. J. Diff. Geom. 9, 435-441 (1974)

8. Hitchin, N., Karlhede, A., Lindström, U., Rocek, M.: Hyperkähler metrics and supersymmetry. Commun. Math. Phys. 108, 535-589 (1987)

9. Kobayashi, S.: Transformation groups in differential geometry. Berlin Heidelberg New York: Springer 1972

10. McInnes, B.: Methods of holonomy theory for Ricci-flat Riemannian manifolds. J. Math. Phys. 32, 888-896 (1991)

11. McInnes, B: Gauge theory in Witten's approach to the generation problem. Commun. Math. Phys. 138, 107-136 (1991) 